\title{
Le judo : une éducation pour faire face à l'incertain ? Le cas des compétitions sans catégories de poids pour les enfants au Japon Learning to face uncertainties through Judo: a study of competitions without
weight categories for children in Japan
}

Thierry Colin • Hélène Joncheray • Misaki Iteya

Résumé : Dans la première moitié du XX ${ }^{\mathrm{e}}$ siècle, l'enracinement du judo en France est accéléré par la représentation qu'il diffuse du plus petit qui vient à bout du plus fort. La motivation des pionniers est portée par l'espoir de l'infini progrès qu'offre le judo et du pouvoir de faire face à toute situation aussi incertaine soit-elle, quelle que soit sa condition. En 1960, avec la montée du sport et la nouvelleorganisation des compétitions prévoyant des catégories depoids, cetespoir s'éteint. La conception philosophique du judo a laissé place à une perspective sportive moins idéalisée. Aujourd'hui, ceci est un fait établi et, de notre point de vue occidental, la compétition sans catégorie de poids de Kan Nai (Japon) pour les enfants se présente comme une pratique étrange en tout point. Elle prend toutefois un sens au Japon puisque depuis la rentrée scolaire 2012, les budô, dont le judô, sont matière obligatoire au collège. Les compétitions sans catégories de poids sont emblématiques d'une éducation que le Japon souhaite réhabiliter, une éducation pour faire face à l'incertain. Nous avons observé cette compétition pour tenter d'en comprendre les conditions de son organisation et sa signification culturelle.

mots clés : judo, enfants, sciences sociales, éducation, incertain.

abstRact: Throughout the first half of the twentieth century, the attractiveness of Judo was accentuated by the ideology it conveyed that the little man could, with work, defeat the big one. The pioneers of judo were motivated by the hope of endless progress and, with it, that of being able to face up to any situation, even the most uncertain - regardless of one's social background. In 1960 however, with the development of this sport and the new organization of competitions now based on weight categories, such hope disappeared. Aless idealized outlook on this activity replaced the philosophical dimension of judo. Today, categories is a given, and from our western point of view, competitions without weight categories, such as the Kan Nai competition for children, seem odd at all levels. Yet they are very meaningful in Japan and since 2012, the budô, including judô, are compulsory teachings in high schools. These teachings then lead to competitions without weight categories that are emblematical of the type of education Japan wishes to promote; an education meant to prepare youngsters to face the uncertainties of life. It is the purpose of this paper to study such competitions in order to understand both their organization and their deep cultural relevance.

KeywoRds: judo, children, social sciences, education, uncertain. 


\section{Zusammenfassung: Judo: eine Erziehung, um mit der Unsicherheit umzugehen ? Das Beispiel der Wettkämpfe ohne Gewichtskategorien für die Kinder in Japan}

In der ersten Hälfte des 20. Jahrhunderts wird die Verbreitung des Judos in Frankreich durch die Vorstellung beschleunigt, dass der Kleine den Starken schlagen kann. 1960 stirbt diese Hoffnung mit der Versportlichung und mit der neuen Wettkampforganisation, die nun Gewichtsklassen vorsieht. Die philosophische Konzeption des Judos weicht einer weniger idealistischen sportlichen Sichtweise. Heute ist dies eine vollendete Tatsache und aus unserem westlichen Blickwinkel erscheint der Wettkampfohne Gewichtskategorienvon Kan nai (Japan) für Kinder eineinjeder Hinsicht befremdliche Praxis darzustellen. In Japan jedoch macht dies Sinn, denn seit dem Schulanfang 2012 sind Budo, und darunter Judo Pflichtfächer in der Mittelschule. Die Wettkämpfe ohne Gewichtskategorien sind kennzeichnend für eine Erziehung, die man in Japan rehabilitieren möchte, eine Erziehung, die dazu dient, mit Unsicherheit klarzukommen. Wir haben diese Art von Wettkampf beobachtet, um seine Organisationsbedingungen und seine kulturelle Bedeutung zu verstehen.

SchlagwöRteR: Judo, Kinder, Sozialwissenschaften; Erziehung, unsicher

\section{Riassunto: Il judo: un'educazione per far fronte all'incertezza? II caso delle competizioni senza categorie di pesi per i bambini in Giappone}

Nella prima metà del XX secolo, il radicamento del judo in Francia è accelerato dalla rappresentazione che è diffusa sul più piccolo che viene ad aver ragione sul più forte. La motivazione dei pionieri è portata dalla speranza dell'infinito progresso che offre il judo e del poter far fronte a ogni situazione per quanto incerta possa essere, quale che sia la sua condizione. Nel 1960, con l'avanzamento dello sport e la nuova organizzazione delle competizioni prevedenti delle categorie di pesi, questa speranza si estingue. La concezione filosofica del judo ha lasciato posto ad una prospettiva sportiva meno idealizzata. Oggi, questo è un fatto stabilito e dal nostro punto di vista occidentale, la competizione senza categorie dipesidi Kan nai (Giappone) peribambini si presenta come una pratica strana in ogni punto. Essa prende tuttavia un senso in Giappone poiché dal rientro scolastico del 2012, i budô, tra cui iljudô, sono materia obbligatoria al college. Le competizioni senza categorie di pesi sono emblematiche di un'educazione che il Giappone auspica riabilitare, un'educazione per far fronte all'incertezza. Noi abbiamo osservato questa competizione per tentare di comprenderne le condizioni della sua organizzazione ed il suo significato culturale.

PaRole chiave: bambini, educazione, incertezza, judo, scienze sociali.

\section{Resumen : El judo : una educación para hacer frente a lo incierto ? El caso de las competencias sin categorías de pesos en los niños de Japón.}

En la primera mitad del siglo 20, el judo se integra en Francia acelerado por una representación que viene del más pequeño hasta el más fuerte. La motivación de los pioneros es la esperanza del infinito progreso que ofrece el judoy poder hacer frente a una situación incierta. En 1960 con lallegada del deporte y una nueva organización de competencias de prevee las categorías de pesos. La concepción filosófica del judo déja el lugar a una práctica deportiva menos idealizada. Hoy en día es un hecho establecido de un punto de vista occidental, la competencia sin categorias de pesos de Kan nai (Japón) de los niños se presenta como una práctica extraña. Esta toma sentido en Japón en el comienzo de la actividad escolar 2012, los budo, y el judo son asignaturas obligatorias en las escuelas. Las competencias sin categorias de peso son emblemáticas en una educación que el Japón desea reestablecer a fin de hacer frente a una educación incierta. Hemos observado esta competencia a fin de entender y comprender las condiciones de su organización y significación cultural.

PalabRas claves : judo, niños, ciencias sociales, educación, incierto. 
Bénéficiant au début du XX $X^{\mathrm{e}}$ siècle d'un courant culturel favorable, le judo se répand très vite en France d'abord sous sa forme d'autodéfense : le jujitsu. À l'époque, la maitrise de cet art venu du Japon garantit mystérieusement à ses adeptesl'avantage del'invulnérabilitéface à l'agresseur quel qu'il soit. Sa redoutable efficacité en fait une arme de choix du plus faible pour se défendre du plus fort. Dans la première moitié du $\mathrm{XX}^{\mathrm{e}}$ siècle, la diffusion du judo en France est portée par la représentation du petit qui renverse le plus fort et la fascination que le Japon opère sur l'Occident.

L'illusion de l'invincibilité s'est toutefois dissipée avec la montée du sport et la réglementation des compétitions en France qui impose des catégories de poids le 22 novembre 1959. Études statistiques à l'appui, la croyance du petit qui renverse le plus grand est un espoir abandonné. Lejudo devient moins un art qu'un sport et perd son idéal. Pourtant, au Japon, pour les adultes comme pour les enfants cette perspective continue d'exister aujourd'hui. À Kan Nai (préfecture de Yokohama), les enfants s'affrontent sans catégories de poids et continuent à faire vivre cette idée qu'il est permis d'espérer fairefaceàtoute situation aussiincertaine soit-elle.

Denotre point devueoccidental, unetelle modalité de rencontre nous semble totalement incongrue. Anti-éducative en France, elle est au Japon précisément des plus formatrices. En effet, dans ce pays les budô, etlejudô en particulier, sont devenus matière obligatoire au collège depuis la rentrée scolaire en avril 2012. On peut saisir davantage le sens d'une telle pratique si l'on considère que la pertinence motrice d'une telle activité est inséparable de sa pertinence sociale. Précisément, la question centrale de notre étude porte sur les conduites motrices mises en jeu, c'est-à-dire les usages, les valeurs, les savoir-faire qui sont mobilisés dans l'affrontement sans catégories de poids et qui prennent un sens dans la société où ils s'appliquent. Le judo pour les enfants au Japon nous intéresse, car il se présente comme une éducation alternative à notre conception pour préparer l'enfant à faire face à l'incertain.

L'altérité des usages éducatifs, employés dans d'autres civilisations ou d'autres temps, grossit par contraste les traits de nos façons de faire et nous renvoie au questionnement sur la signification de notre pratique. Pour cette raison, nous exploiterons la comparaison dans notre démarche d'étude.

\section{1. le Petit qui RenveRse le Plus gRand : du mystique au scientifique Jusqu'à l'imPRobable}

\subsection{L'origine mystique du jujitsu}

Le 26 octobre 1905, à Courbevoie, le combat du lutteur Georges Dubois contre le spécialiste de la méthode japonaise Ernest Régnier est l'événement historique qui va populariser le judo en France sous sa forme d'autodéfense. L'étonnement vient de la différence de force apparente entre les deux combattants et de la vitesse à conclure le duel. Contre toute attente, six secondes d'affrontement suffisent à Régnier, $63 \mathrm{~kg}$, pour provoquer l'abandon du célèbre lutteur de $75 \mathrm{~kg}$.

Cette rencontre dépasse le cadre de l'affrontement de deux hommes et s'étend à celui de deux cultures, dont on compare les réussites artistiques, militaires et économiques. Le Japon, si petit géographiquement, étonne par sa capacité à rivaliser avec des puissances étrangères affirmées et devient figure emblématique du petit qui vient à bout du plus fort. L'inintelligibilité partielle de cette réussite conduit à un culte irraisonnépourlejujitsu.

« Doctrine insolite du corps, le judo possède une altérité absolue et fascinante qui rend l'ailleurs del'Orientaccessible. [...] C'estle prisme de l'exotisme qui donne à la technique son apparente supériorité culturelle " (Brousse, 2005, p. 20).

\subsection{Une approche scientifique du judo}

Dans les années 1930, le judo est à présent moins apprécié comme un sport de défense 
qu'une éducation physique et morale. Une perception moins mystique que scientifique se dessine de son efficacité. Le principe fondamental du judo énoncé par Jigorô Kanô, qui réside dans l'utilisation optimale de son énergie (seiryoku zenyo) va séduire dans les années 1940 de nombreux universitaires et éminents scientifiques tels qu'Irène Joliot-Curie ou Frédéric Joliot, qui voient dans l'application rationnelle et travaillée des lois de la biomécanique un moyen de renverser la hiérarchie naturelle établie par la différence de force entre individus. Leur motivation est portée par l'espoir investi dans la technique associée à la science capable de dépasser l'ordre imposé par la nature et qui trouve son application dans les affrontements en judo sans catégorie de poids. Le mystique laisse la place au rationnel.

\subsection{Les catégories de poids et la " désacralisation du judo " (Brousse, 2005)}

L'institutionnalisation rapide du judo sportif dans la seconde moitié du $\mathrm{XX}^{\mathrm{e}}$ siècle, conduira la fédération de judo à adopter dans les années 1950 les catégories de poids dans les compétitions, au terme d'un long débat entre partisans du mouvement sportif et traditionalistes, quivoient dans cetteévolution la perversion d'une pratique d'abord appréciée comme un art. Dans une démarche plus pragmatique que philosophique, statistiques à l'appui, on écarte l'hypothèse que l'expertise en judo permettedefairefaceàtoutadversaireencompétition quel que soit son poids. L’idéal de progrès s'efface et le judo s'affranchit de cette glorieuse ambition d'offrir à chacun quelle que soit sa condition le pouvoir de faire face à l'incertain.

C'est une concession historique du judo au mouvement sportif qui ne pouvait s'accommoder des rencontres sans catégories de poids. La montéedusportgénèredestransformationsdes pratiques et des usages. Selon Pierre Parlebas
(1999, p. 85), « le sport correspond à l’institutionnalisation de contraintes motrices qui éliminent le désordre et la confusion ». Pour cette raison, « les conditions du duel se présentent comme un modèle de symétrie et d'équilibre ».

La constitution de catégories d'âge, de poids et de sexe permet d'assurer l'égalité des chances de tous les combattants conformément àla morale sportive.

Une page de l'histoire du judo se tourne. La période d'enracinement s'achève avec « le début d'une étape de large diffusion accompagnée d'un renouvellement de génération et d'un profond changement de valeur avec l'influence croissante du modèle sportif » (Brousse, 2005, p. 130).

À partir de cette date, le judo va fidéliser un nombre croissant d'enfants toujours plus jeunes. Sans doute est-ce là le phénomène le plus marquant de cette deuxième page de l'histoire du judo en France.

\section{2. le Judo des enfants en fRance et au JaPon, auJouRd'hui}

\subsection{Des similitudes et une différence majeure entre les deux méthodes}

En France, le judo des enfants va se développer avec une référence forte au Japon. Les similitudes entre les deux méthodes sont nombreuses. Tout d'abord, les deux méthodes ont la même référence historique au fondateur Jigorô Kanô et à sa définition éducative du judo. Cette orientation partagée est confirmée dans notre questionnaire distribué aux parents et professeurs de judo des deux pays en 2009 (Colin, 2010, p. 62). À la question des finalités du judo des enfants, la réponse est unanime : « recevoir une éducation du corps et de l'esprit » ${ }^{1}$ età la question des compétences, ils choisissent ensemble: «l'apprentissage desrèglessociales » ${ }^{2}$.Une

1 Àla question «Quelles finalités pour la pratique du judo des enfants?» professeurs et parents japonais sont $60 \%$ en moyenne à choisir comme premier choix de réponse « Recevoir uneéducation du corps et de l'esprit » et $52 \%$ pour les professeurs et parents français.

2 À la question "Quelles compétences est-il nécessaire de développer chez l'enfant par le judo ?" professeurs et parents japonais sont $37 \%$ en moyenne à choisir comme premier choix de réponse «Les règles sociales (politesse et respect) » et $41 \%$ pour les professeurs et parents français. 
autre similitude tient à la distance prise avec le sport et l'adoption d'un règlement adapté des combats assez proche dans sa définition. Enfin, les deux pays partagent la même conception du style de judo à enseigner aux enfants, appelé au Japon le tadashiijudô (le judojuste).

Lejudo des enfants français semble en tout point être celui des enfants japonais. En France, dans les représentations collectives, l'apprentissage du judo reproduit une éducation japonaise idéalisée. La mondialisation du judo parait avoir uniformisé la pratique. Toutefois, les compétitions organisées sans catégories de poids pour les enfants au Japon se présentent de notre point de vue occidental comme une bizarrerie. Ce qui, dans la première moitié du $\mathrm{XX}^{\mathrm{e}}$ siècle, donnait en France son sens au judo est à présent perçu comme une étrangeté qui repousse soudain loin de nous la pratique japonaise. Cette modalité d'affrontement intéresse notre sujet, car elle place l'enfant japonais dans une situation de grande incertitude, probablement insoutenable pour laplupart des enfants français.

Au cours d'un séjour d'étude à l'Université de Tokyô Gakugei, de septembre 2008 àjuillet 2009, nous avons observé une telle organisation à Kan Nai (préfecture de Yokohama) le 12 avril2009, où s'affrontaient des enfants de 7 à 12 ans réunis par âge, mais sans distinction de sexe et de poids. 623 participants, 604 combats dont nous avons statistiquement relevéles résultats en considérant le poids de chacun. Filles et garçons s'affrontent avec des écarts de poids pouvant atteindre, certaines fois, $50 \mathrm{~kg}$ et plus. Malgré le risque que cela représente, la sécurité des enfants ne semble jamais inquiétée. L'organisation sans catégorie de poids constitue manifestement une singularité culturelle, un trait ethnomoteur pour nous difficilement compréhensible, si l'on ne fait pas référence à Marcel Mauss (1950). Cet auteur l'a montré, la motricité est enchâssée dans une logique sociale. Le combat justifie une préparation physique adaptée, mais également l'intériorisation de principes et de valeurs qui sont autant d'habitus qui vont régler les usages de l'affrontement et plus durablement les attitudes sociales. Les techniques du corps (Mauss, 1950) en judo et l'organisation des affrontements prennent un sens dans le contexte social où elles sont appliquées.

\subsection{La compétition de judo sans catégories de poids de Kan Nai (préfecture de Yokohama)}

À Kan Nai, ce jour-là,l'organisation de la manifestation est parfaite. À $9 \mathrm{~h} 00$ précises, les enfants sont alignés sur le grand tatami etécoutent les discours des anciens, appelant notamment à combattre en appliquant un style de judo juste (tadashii judô). Puis, chacun se tourne vers le drapeau du pays, l'hymne japonais est entendu. Nous nous procurons un programme sur lequel figurent les tableaux de compétition pour chaque âge et pour chaque enfant, révélant de nombreuses informations précieuses pour notre étude : le poids, la taille, gaucher ou droitier, et un commentaire de l'enfant lui-même. Les tableaux de compétitions affichés et régulièrement complétés nous donnent les résultats de tous les combats. Ce jour-là, le vainqueur de la catégorie des enfants de 8 ans $\left(2^{\mathrm{e}}\right.$ année scolaire) n'est pas le plus lourd. C'est un garçon qui pèse $24 \mathrm{~kg}$ et qui a éliminé successivement des adversaires de 24, 56, 22, 42, 30 et $38 \mathrm{~kg}$.

À partir des informations collectées tout au long de la journée, nous confrontons la différence de poids des combattants et le résultat de leur rencontre (figure 1). Sur l'ensemble des rencontres des six catégories d'âge, 133 combats sont gagnés par lejudoka le plus léger, soit 33,4\% de l'ensemble des combats. La différence de poids entre les deux combattants excède alors plus de $10 \%$ du poids de corps du plus léger. 202 combats sont gagnés par un judoka dont la différence est comprise entre- $10 \%$ et $+10 \%^{3} .268$ combats sont gagnés

3 Il s'agit dans ce cas d'une situation semblable à celle qui est le plus souvent organisée en France. En effet, on admet habituellement que la différence de poids entre les deux combattants ne doit pas excéder $10 \%$ du poids du corps du plus léger. 
Figure 1. Résultats des combats de la compétition de Kan nai (Japon) selon la différence de poids des enfants. Exemple d'interprétation: 25 enfants ont gagné alors que le poids de leur adversaire était plus important. La différence de poids (-30\%) était comprise entre 21 et $30 \%$ du poids de corps du plus léger.

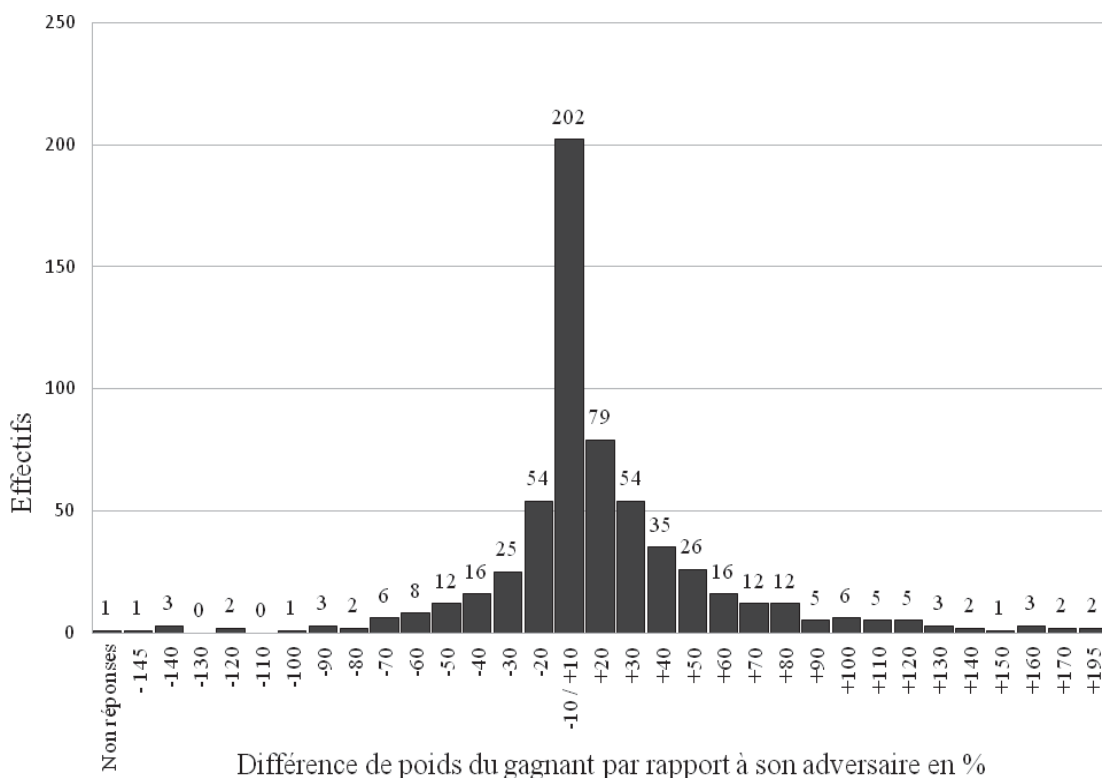

par des combattants plus lourds et dont la différence de poids excède $10 \%$. L'avantage du poids semble confirmé. Toutefois, les judokas plus légers sont également une proportion importante à gagner leurs combats. Il est remarquable de constater qu'ils sont encore 38 à gagner lorsque la différence de poids de corps qui leur est défavorable est supérieure à $50 \%$. Des valeurs suffisantes pourquel'espoir du petitcapable de renverser le plus lourd puisse continuerà entretenirl'enthousiasme desenfants.

\subsection{La gestion de l'incertain en judo en France et au Japon}

En raison de sa nature, le combat de judo place l'enfant dans une situation d'incertitude qui tient à l'adversaire. La difficulté de la tâche réside moins dans le geste technique à maîtriser que dans son adaptation à l'imprévisibilité du mouvement de son opposant. Toutefois, alors qu'en France l'organisation tend à définir au plus près le cadre de la pratique pour l'enfant et à satisfaire immédiatement son expression, au Japon elle semble au contraire entretenir l'incertitude.

À Kan Nai, avant d'avoir le programme de la compétition, l'enfant ne peut prévoir s'il combattra face à une fille ou un garçon ; il ne connaît pasnon pluslepoids etlataille deson futur adversaire. Le moment de son premier combat est également incertain et sa compétition peut s'arrêter tôt le matin en quelques secondes dès le premier combat, s'il est rapidement projeté avec efficacité (projection comptabilisée Ippon) ou se poursuivre très tard dans la journée s’il parvient au stade final. En cas de défaite, aucune seconde chance ne lui est offerte. Pour l'enfant japonais, il ne sera pas simple de se distinguer, car le nombre de participants est important. Dans la catégorie d'âge des 11 ans ( $5^{\mathrm{e}}$ année scolaire) et des 12 ans ( $6^{e}$ année scolaire), ils sont respectivement 132 et 131 combattants.

Différemment en France, il est généralement admis que la différence de poids entre les enfants ne dépasse pas $10 \%$ du poids de 
corps du plus léger. Le protocole prévoit généralement que l'animation ne s'étale pas sur une durée de plus de deux heures. Quelle que soit la qualité de sa prestation, l'enfant sait qu'il aura deux combats à disputer et une épreuve de démonstration complice avec un partenaire. Dans ces trois épreuves, l'enfant accumule des points et se situe en conséquence dans une des trois catégories bronze, argent, ou or. Quelle que soit sa prestation, il est classé et médaillé.

Au Japon, l'incertitude maintenue rend la tâche difficile et la possibilité de briller par son résultat très peu probable. Le vide laissé par le cadre de l'organisation japonaise contraste avec le plein du modèle français dont le souci est de réduire au mieux l'incertitude spatiale, temporelle et événementielle, afin de donner satisfactionimmédiateà chacun desparticipants.

\subsection{Nature contre culture}

Entre le modèle japonais et le modèle français, ce sont deux contextes différents d'expression qui sont présentés et l'éducation de deux représentations différentes de la rencontre. Dans la compétition, toutes catégories de poids confondues, l'enfant apprend à agir dans un cadre incertain. C'est également le lot de sa condition de vie géographique et sociale. En effet, les Japonais subissent régulièrementles rigueurs d'une nature sujette aux cataclysmes, dont les séismes qui ont dévasté Tôkyô en 1923 (130 000 morts), Kôbe en 1995 (5478 morts) et plus récemment Fukushima (15 700 morts et 4700 disparus) ne sont que les derniers exemples en date.

Dans lecombat en judo se rejoueà chaque fois le duel déséquilibré d'une nature hostile et imprévisible, dont les Japonais ont hérité, et d'une technique patiemment travaillée. L'enfant le plus lourd, généreusement doté par la nature rencontre un enfant plus léger qui peut être en mesure de renverser l'ordre hiérarchique naturel avec les moyens reçus en héritage culturel. Le dojo est le théâtre d'une rencontre à l'issue incertaine selon une mise en scène qui magnifie la culture nippone en suscitant une grande émotion. Depuis le Japon, les rencontres internationales sont fréquemment jugées comme peu attrayantes. Le président de la fédération japonaise de judo, Haruki Uemura, le rappelait lui-même dans un entretien avec le professeur Ogawa, le 24 avril 2009: "Comme le judo est devenu un sport mondial, il n'est plus une partie de la culture du Japon, mais c'est une culture du Judo enracinée dans le monde. Dans ce processus, des problèmes ont inévitablement surgi. Certains disent que le Judo est devenu très étrange, peu intéressant.»

\section{3. les conditions de l'affRontement sans catégoRies de Poids des enfants}

Les conditions de l'affrontement, c'est-àdire les usages, les règles, les normes, les valeurs requises sont tout à la fois ce qui va permettre le combat dans des conditions déséquilibrées et ce qui constitue les fondements de la socialisation de l'enfant. La méthode japonaise séduit par ses résultats cependant les conditions de sa mise en œuvre semblent inconcevables en France. Comment unetelle organisation estelle possible au Japon sans menacer l'intégrité physiqueetla motivation des enfants?

Les combats sans catégories de poids le plus souvent d'une grande intensité se déroulent sans agressivité incontrôlée apparente. Les caractères essentiels du style japonais qui semblent autoriser une telle confrontation nous semblent résider principalement dans la nature de la leçon de judo, l'étiquette (reigi) et le style : le tadashii judo (judo juste).

\subsection{La leçon au Japon}

Au Japon, l'intensité et la fréquence hebdomadaire des leçons sont variables suivant les clubs. Toutefois, les enfants judokas japonais s'entraînent en moyenne deux fois par semaine entre 7 et 9 ans contre une fois pour les Français et quatre à cinq fois en moyenne par semaine entre 10 et 12 ans, contre deux fois pour les Français (Colin, 2010). Dans la séance, lapartimportantedes exercices depréparation 
physique générale (sauter, courir...) et la répétition systématique des exercices d'apprentissage des chutes préparent l'enfant à subir la contrainte d'un adversaire plus lourd dans un combat. Par ailleurs, les qualités de persévérance sont très valorisées et éprouvées dans les longues séquences de répétition du geste technique. Dans notre enquête auprès des professeurs et des parents japonais et français, à la question des attitudes à développer, la préférence des Japonais va au développement " de la capacité à répéter longuement pour apprendre... la pugnacité, la combativité... la résistanceà l'effort physique et moral » (Colin, 2010, p. 63) ${ }^{4}$.

Préparation physique et technique offrent à l'enfant des gestes offensifs d'une vitesse et d'une précision quiétonnent souvent lesétrangers. Ces conditions de préparation sont déjà de nature à préparer le corps des enfants à la rencontre.

\subsection{Le reigi}

Observer l'étiquette consiste d'abord à appliquer un salut parfaitement exécuté. Nombreux sont les grands champions à être invités dans les compétitions pour détailler et commenter le salut. Il est aussil'élément central du projet "renaissance du judo au Japon " adopté par la fédération de judo japonaise en 2001, qui vise à réhabiliter les valeurs traditionnelles du judo. Le rituel du salut, ce geste en apparence si insignifiant, semble symboliser à lui seul tout le sens et l'enjeu de l'éducation sociale par le judo au Japon. Selon J. Kanô, " le jûdô kôdôkan commença, composé de trois grosses mailles qui étaientl'entrainement physique, la formation mentale et l'entrainement au combat. [...] Les années 1915-1925 voient l'apparition d'une quatrième maille : l'application des principes dans la société. L'étiquette (reigi) entre alors dans la catégorie d'éducation morale. [...] En février 1915, Kanô Jigorô définit temporairement le but du judo comme étant "se réaliser et contribuer à la société" " (Cadot, 2008, p. 83).

Les combats sans catégories de poids donnent lieu quelquefois à des affrontements très intenses. Le salut de leur adversaire, que les enfants s'appliquent à réaliser religieusement avant et après l'affrontement, se présente comme un moment d'apaisement de la relation. Pour le réaliser, d'abord, l'enfant se redresse comme le maître lui demande pendantl'entraînement: «kio tsuke » (prendre une bonne posture et un état d'esprit concentré), puis annonce dans son mouvement d'inclinaison du buste: " onegai shimasu » (invitation à travailler ensemble) en début de combat et " arigatô gozaimasu " (merci beaucoup) àl'issue du combat. Il s'avance ensuite vers le maitre et le salue en position agenouillée avant d'écouter son commentaire du combat.

Le salut est un geste qui rythme aussi les leçons de judo. Il se réalise suivant des rituels multiples selon qu'il soit fait seul ou en groupe, en début ou en fin de séance. Chaque détailde sa réalisation compte : l'inclinaison, la place occupée, et révèle son rang dans la hiérarchie sociale. Collectivement réalisé, il rappelle l'adhésion au groupe et à ses valeurs. Le rituel du salut est un élément fort de la tradition du judo que le Japon soigne particulièrement, revendiquant « lejudo d'origine » où les petits gestes que nous, occidentaux, nommons vides, sont, pour eux, les plus pleins de tous.

\subsection{Le tadashiijudo}

À Kan Nai, Kaori Yamaguchi première championne du monde japonaise à Vienne en 1984, donne une leçon de judo sur un tatami laissé libre pour les enfants qui sont en attente de combattre. Son intervention porte sur les éléments traditionnels du judo, le reigi et le tadashii judô.

Détaillons quelques-unes des particularités du tadashii judo. La saisie classique tout d'abord. Dans le combat, la saisie est déterminante, elle

4 Àlaquestion «Quellesattitudes est-ilnécessaire de développerchezl'enfantparlejudo? », la réponsecitée regroupeen moyenne64\%des premiers choix des Japonais alors qu'elle ne totalise en moyenne que $26,25 \%$ de ceux des Français. 
prépare l'action offensive. Globalement, deux styles peuvent être discernés, même si la réalité apporte plus de nuances. Pour l'une, la saisie se fait davantage dans le dos del'adversaire. L'intention est de peser de toute sa force, de « plier » le concurrent, afin de le soumettre à son action de projection. Dans l'autre style, qui représente l'école japonaise, la main qui saisit le revers (tsurite) reste sur le devant du judogi de l'adversaire. Le rôle de la saisie est dans ce cas de contrôler le mouvement de son opposant. Les mains sont tour à tour de fins capteurs qui détectent le mouvement adverse afin de l'anticiper, et de puissants transmetteurs de l'action. Elles sont l'instrument du dialogue plus qu'un outil de tentative de soumission adverse. Cette particularité permet d'installer la relation dans le combat non pas sur le principe de la force, mais plutôt de la bonne utilisation de l'énergie de l'adversaire et permet en cela la rencontre sanscatégories depoids.

Ce second style de saisie qu'affectionnent les Japonais a un autre rôle. Il permet au judoka de garder son adversaire à distance. L'espace conservé entre les deux judokas est exploité par l'attaquant pour s'élancer dans la réalisation d'une technique de grande amplitude qui sera comptabilisée Ippon, c'est-à-dire maximale dans son efficacité, et en outre dans sa spectacularité.

Le respect de l'étiquette, le caractère vulgaire de l'emploi de la force, l'attitude redressée, la recherche de la victoire avec l'emploi d'une technique décisive font référence aux valeurs et à la technique du samouraï, figure emblématique du Japon, et assure à celui qui reproduit le style tadashii judo l'illustre noblesse d'âme des guerriers nippons de l'avant meiji. Le judo juste se double d'une valeur esthétique et sociale, qui fait référence à la tradition martiale du Japon.

"On parle parfois d'un "vrai judo". Mais il est difficile de le définir. Il faudrait chercher plutôt un "beau judo". Voiciquelques éléments de la beautédu judo: les deux adversaires se saisissent avec le hikite (saisieà la manche) etletsurite (saisieau revers), un kumite (la saisie) naturel sans plier le corps, tenter des techniques qui permettent de gagner par ippon, maintenirl'espritoffensif, etc. Il peuty avoirun beau judo même quand on est battu » (K. Yamaguchi, citée par Ogawa, 28 mars 2009).

Aujourd'hui, le judo pour les enfants au Japon est une expression culturelle dominante puisque les budô, et le judô en particulier, ont été inscrits officiellement comme matière obligatoire au collège à la rentrée scolaire en avril 2012.

\subsection{Les budô dans les programmes scolaires au collège}

Les élèves doivent pratiquer un budô parmi judô, kendô, sumô et karaté en $1^{\mathrm{re}}$ et $2^{\mathrm{e}}$ année de collège. En $3^{\mathrm{e}}$ année ils choisissent un budô et un sport de balle. Chaque établissement retient un des budô suivant la qualification de ses professeurs, les installations disponibles... Avec un volume horaire annuel obligatoire relativement faible, 13 heures, il n'est pas attendu que la pratique transforme physiquement et techniquement les enfants, mais plutôt qu'elle leur enseigne l'étiquette, la politesse, le respect du partenaire (Satô, Samejima, \& Takahashi, 2009), autant d'éléments fondamentaux de la tradition japonaise, et notamment du confucianisme, qui enseignent l'abnégation, la piété filiale, l'harmonie des relations humaines, la politesse formalisée. Les budô participentà un mouvement de rénovation du système éducatif sur lequel le Premier ministre Koizumi Junichirô avait déjà beaucoup travaillé de 2001 à 2006. Après lui, cet effort a aboutien décembre 2006 à la modification de la loi fondamentale sur l'éducation de 1947 (kyôiu kihonhô). Aujourd'hui, c'est dans le passé que le Japon semble trouver le moyen de faire face à un avenir incertain.

\section{Conclusion}

Le caractère étranger, sinon étrange, del'organisation des compétitions sans catégories de poids de Kan Nai nous interpelle. Nous observons des combats de grande intensité sans que 
la sécurité de l'enfant, à laquelle les Japonais sont très attachés, soit négligée. Nous n'observons pas de blessure. Quelles motivations les enfantsjaponais trouvent-ils ce jour-là pour combattre dans des conditions très imprévisibles et pour un titre presque illusoire (623 enfants et 6 titres)? On comprend mieux le sens de cette pratique si l'on fait référence aux principes du confucianisme présents dans l'enseignement du judo, qui enseignent l'abnégation, le respect d'autrui et la piété filiale, si l'on perçoit la portée esthétique du geste en référence aux prestigieux samouraïs, si l'on est sensible à la valeur spectaculaire d'une lutte symbolique de la nature hostile face à la culture nippone magnifiée, et enfin sil'on sait l'attachement politique contemporain aux traditions nippones. Ce ne sont là que quelques éléments du contexte qui donnent du sens au combat sans catégories de poids des enfants. L'analyse diachronique ou interculturelle des pratiques motrices nous révèle des modèles originaux pour faire face au contexte changeant et imprévisible de notre société. Notre collaboration avec le Japon se présente comme un puissant élément d’inspiration et de stimulation pour l'innovation éducative. Nous souhaitons que l'échange d'idées ne conduise pas à l'uniformisation des pratiques. "Au contraire, qu'il s'agisse d'éducation, de fête ou de culture, les identités sont à préserver, puisque c'est à partir d'elles, et non dans leur négation, que se développent les communications internationales. La coopération des peuples ne suppose pas seulement l'existence des moyens de communication, comme sont le sport ou certaines langues : encore faut-il que chacun ait quelque chose à dire, qui témoigne de son identité " (During, 2007, p. 643)5.

\section{bibliogRaPhie}

bRousse, m. (2005). Les racines du judo français. Bordeaux, Presses universitaires de Bordeaux.

Cadot, y. (2008). Le salut chezles jûdôkas français du point devue des études sur Kanô Jigorô, in National Institute of Fitness and Sports in Kanoya (Ed.), International bûdô symposium report. The mind of martial arts: rules of decorum (pp. 82-89). Kagoshima, Japon, 7 décembre.

colin, t. (2010). Analyse sociologique comparative de la pratique du judo pour les enfants de sept à douze ans au Japon et en France. Thèse de doctorat non publiée, Université Paris V René Descartes, La Sorbonne, Paris.

duRing, b. (1997). Jeux fêtes et culture: quelques axes d'analyse, in F. Amador Ramírez, U. Castro Núñez \& J.M. Álamo Mendoza (Eds.), Luchas, deportes de combate y juegos tradicionales (pp. 631-643). Las Palmas de Gran Canaria, Gymnos Editorial Deportiva.

mauss, m. (1950). Les techniques du corps, in M. Mauss (Ed.), Sociologie et anthropologie, Paris, Presses universitaires de France, pp. 363-386.

ogawa, g. (2009). Haruki Uemura, nouveau leader du judo Japon, explique ses intentions. Entretien non publié, Site internet http://www.judo-voj.com.

PaRlebas, P. (1981/ 1999). Jeux, sports et sociétés. Lexique de praxéologie motrice. Paris, Insep.

Satô, y., Samelima, m., \& taKahashi, K. (2009). Enseigner le judo au collège avec efficacité et sécurité, préparer l'obligation des budô (en japonais). Judô, janvier, Tokyô,Japon, Kôdôkan, 89-91. 